\title{
ArcheoSciences
}

Revue d'archéométrie

\section{Apport de la géostatistique à la connaissance de la morphologie naturelle du site de Bordeaux (France)}

Geostatistical contribution to the knowledge of the natural morphology of Bordeaux (France)

Sophie Dominique, Antoine Marache et Pierre Régaldo-Saint Blancard

\section{OpenEdition}

\section{Journals}

Édition électronique

URL : http://journals.openedition.org/archeosciences/2598

DOI : $10.4000 /$ archeosciences. 2598

ISSN : 2104-3728

\section{Éditeur}

Presses universitaires de Rennes

\section{Édition imprimée}

Date de publication : 10 avril 2010

Pagination : 25-37

ISBN : 978-2-7535-1407-2

ISSN : $1960-1360$

\section{Référence électronique}

Sophie Dominique, Antoine Marache et Pierre Régaldo-Saint Blancard, « Apport de la géostatistique à la connaissance de la morphologie naturelle du site de Bordeaux (France) », ArcheoSciences [En ligne], 34 | 2010, mis en ligne le 10 avril 2013, consulté le 19 janvier 2021. URL : http://

journals.openedition.org/archeosciences/2598; DOI : https://doi.org/10.4000/archeosciences.2598 


\title{
Apport de la géostatistique à la connaissance de la morphologie naturelle du site de Bordeaux (France)
}

\author{
Geostatistical contribution to the knowledge \\ of the natural morphology of Bordeaux (France)
}

\section{Sophie Dominique*, Antoine Marache* et Pierre Régaldo-Saint Blancard **}

\begin{abstract}
Résumé : En site urbain, à la base des niveaux anthropisés, le paléorelief correspond globalement à la surface géologique naturelle la plus récente. La connaissance de cette surface est capitale pour comprendre les raisons et les modalités des implantations humaines (accessibilité et position du site par exemple). L'objectif poursuivi est d'utiliser les données de forages disponibles dans le cœur historique de Bordeaux, pour les intégrer dans un modèle 2,5D. L'un des éléments majeurs de cette problématique est la localisation et l'évolution des cours des deux principales rivières du centre ville que sont le Peugue et la Devèze. Un modèle a été construit sur le centre de Bordeaux, par krigeage sous contraintes d'inégalités en utilisant principalement les données litho-stratigraphiques des sondages géotechniques. Celui-ci permet de voir sous un jour nouveau l'évolution géomorphologique du centre ville historique et de montrer que l'image qu'avaient les archéologues sur l'hydrographie de ce secteur était incomplète.

Abstract: In urban location, paleorelief, below the anthropogenic surface, is the most recent natural geological layer. In order to understand the reasons and modalities of human implantations (site accessibility and location for example), it is capital to develop the knowledge of this surface. We have a database gathering many boreholes done in Bordeaux historical downtown. This database has been used for building a 2.5D model. A main question is the location and the evolution of the two main rivers of the town, the Peugue River and the Deveze River. This model has been built on Bordeaux town centre by using kriging under inequalities constraints provided by the litho-stratigraphic data of geotechnical boreholes. This model provides an innovative point of view about the geomorphological evolution of the town centre. It also shows that the representation the archaeologists had about historical evolution of the hydrography was incomplete.
\end{abstract}

Mots clé : archéologie urbaine, modélisation, sondages géotechniques, géostatistique.

Key words: urban archaeology, modelling, geotechnical boreholes, geostatistics.

\section{INTRODUCTION}

Les problématiques archéologiques de naissance et de développement des villes sont étroitement liées à la recon- naissance dans le sous-sol de l'interface entre les couches naturelles et anthropisées, interface correspondant à la topographie du site "naturel " lors de la première phase d'occupation. Lors du développement de la ville, plusieurs

\footnotetext{
*Université de Bordeaux, GHYMAC - Av. des facultés, 33405 Talence cedex, France. (s.dominique@ghymac.u-bordeaux1.fr), (a.marache@ghymac.ubordeaux1.fr)

** DRAC Aquitaine, SRA - 54 rue Magendie, 33074 Bordeaux cedex, France. (pierre.regaldo@culture.gouv.fr)
} 
mètres de matériaux ont été déposés depuis cette topographie du site « naturel " (mur des couches anthropisées ou toit du substratum tertiaire dans notre cas) jusqu'à la surface topographique actuelle (Galinié et al., 2003). Ces matériaux, qualifiés de dépôts anthropiques, correspondent le plus souvent aux remblais des géotechniciens qui les définissent par la présence de débris de matériaux divers. On cherche ici à reconstruire cette première interface discordante ainsi que celle qui sépare le substratum tertiaire des dépôts alluvionnaires quaternaires.

Ce travail a été conduit dans le cadre du projet RIVIERA "RIsques en VIlle : Equipements, Réseaux, Archéologie " qui vise au développement de méthodes et d'outils pour l'évaluation préalable des " aléas d'origine géotechnique " à l'échelle d'une agglomération. Ses applications portent sur la communauté urbaine de Bordeaux et, pour ce qui concerne l'archéologie, le centre historique de la ville.

Bordeaux est traversé d'ouest en est par deux rivières affluentes de la Garonne : la Devèze et le Peugue. La première, avec un cours de $10 \mathrm{~km}$, est aujourd'hui conçue comme un affluent du second, long de $13 \mathrm{~km}$; leurs affluents principaux, le Caudéran et le ruisseau des Ontines, mesurent respectivement $4 \mathrm{~km}$ et $6 \mathrm{~km}$; le bassin versant de ce réseau a une superficie maximale de $25 \mathrm{~km}^{2}$. Effacées du paysage actuel par des ouvrages souterrains, elles coulaient, depuis les premières attestations médiévales, de part et d'autre de la même vallée et étaient soumises à un régime intertidal dans tout le cœur de la ville. C'est au nord, sur le plateau découpé par la Devèze, que s'est développée, à partir d'une présence humaine plus ou moins sporadique au Néolithique et à l'âge du Bronze, dès le $\mathrm{VI}^{\mathrm{e}}$ siècle avant notre ère, une agglomération protohistorique (Lavaud [dir.], 2009). L'expansion progressive de la ville romaine qui en naît ne semble franchir la vallée que vers le début du $\mathrm{II}^{\mathrm{e}}$ siècle. A la fin du $\mathrm{III}^{\mathrm{e}}$ siècle, une restructuration urbaine radicale établit une puissante enceinte autour du port intérieur installé sur la Devèze. Le Peugue, quant à lui, longe cette enceinte au sud; vers le $\mathrm{XI}^{\mathrm{e}}$ siècle, il accueille à son tour un port (Lavaud [dir.], 2009).

L'existence ancienne des deux rivières est un présupposé, issu du constat de leur présence durant le dernier millénaire, qui s'est implicitement imposé aux historiens de la ville (Etienne, 1962). Plusieurs découvertes archéologiques des dernières décennies viennent à l'encontre de cette idée. En 1981, les fouilles de l'îlot Saint-Christoly ont mis en évidence la confluence avec la Devèze d'un petit cours d'eau venant du sud, qui disparaitrait vers la fin du III $^{\mathrm{e}}$ siècle, que l'on identifie au Peugue (Debord et Gauthier, 1982). Un peu en amont, environ $150 \mathrm{~m}$ au sud-ouest, a été fouillée, sur le versant sud d'un fond de vallée progressivement colmatée à partir du milieu du $\mathrm{I}^{\mathrm{er}}$ siècle, la culée d'un pont antique qui devait franchir le même cours d'eau (Sireix dir., 1995, 2008). Encore $150 \mathrm{~m}$ en amont, une fouille menée en 2009, trop récente pour être publiée, a retrouvé un paléochenal correspondant sans doute au même bras de rivière, possédant un atterrissement en bois et un mur de quai, actif durant le Haut Empire et progressivement abandonné à partir de la fin du $\mathrm{II}^{\mathrm{e}}$ siècle. Enfin, pour certains - mais ce point de vue n'a pas été adopté par tous -, le Peugue aurait été détourné, de sa position initiale de convergence avec la Devèze pour son cours connu au Moyen Age, lors de la construction du rempart antique (Barraud et al., 1996).

Tout au début des recherches de RIVIERA, à titre d'hypothèse de départ, a été proposé un schéma général (RégaldoSaint Blancard, 2004 [2009]) qui tenait compte à la fois de l'ensemble des faits observés et de tous les raisonnements tenus. Il peut se résumer en une évolution en cinq temps : convergence des deux rivières à l'ouest de Bordeaux avant l'époque flandrienne; distinction des deux lits jusqu'au début de l'époque romaine; organisation d'une confluence à Saint-Christoly vers 70 ; report de cette confluence à l'extérieur du rempart à l'extrême fin du III e siècle, avec la plausible exploitation d'un bras du Peugue préexistant, contemporain de celui attesté à Saint-Christoly; envasement de la Devèze à l'ouest des remparts de la ville à partir du VI ${ }^{\mathrm{e}}$ siècle et développement d'une zone marécageuse, d'où une partie des eaux (le Peugue) se fraye un débouché par le sud.

Après avoir fait l'inventaire des informations disponibles, on montrera comment un traitement géostatistique de ces données disponibles permet de visualiser et de comprendre la mise en place de la morphologie naturelle du site de Bordeaux.

\section{DONNÉES DISPONIBLES}

C'est le cour historique de Bordeaux qui, en raison de sa richesse en investigations archéologiques, a fait l'objet de la modélisation de l'interface sol anthropique/sol naturel. Les archéologues en ont une image "a priori " très complète, qui peut être facilement comparée aux résultats des cartes obtenues par interpolation à partir des données litho-stratigraphiques.

\section{Utilisation des sondages géotechniques}

Sur ce secteur du centre-ville, d'une étendue d'environ $2 \mathrm{~km}^{2}$, on dispose de 461 sondages issus de diverses campagnes de forages (fig. 1) délivrant une information lithologique complète mais hétérogène dans sa description. Après transcription de ces données par le biais d'un référentiel 
Figure 1 : Localisation des données utilisées. Figure 1: Location of used data.

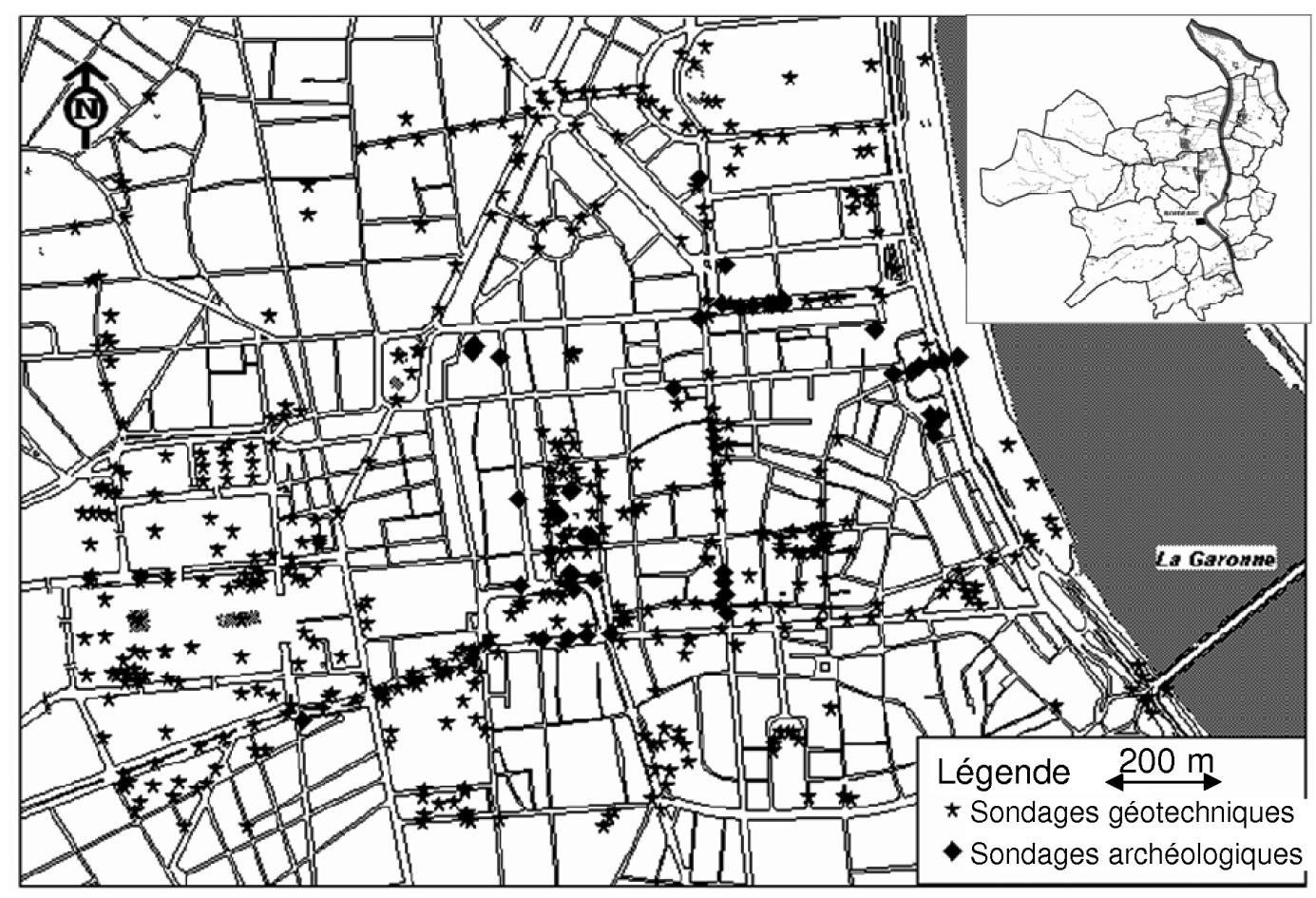

(Thierry et al., 2006) permettant de décrire au mieux les terrains rencontrés tout en homogénéisant les différents vocabulaires, nous avons traduit ces informations sous forme de trois ensembles stratigraphiques (fig. 2) :

- l'Anthroposol, dépôts anthropiques pour l'archéologue ou remblais pour le géotechnicien;

- le Quaternaire, regroupant à la fois les formations Flandriennes (correspondant à l'alluvionnement de la Garonne durant l'Holocène) et les formations des terrasses fluviatiles (Pléistocène) de la Garonne;

- le Substratum, daté de l'Oligocène $(23.5 \mathrm{Ma})$ dans le secteur modélisé, constitué principalement de formations marno-calcaires.

\section{Emploi des données archéologiques}

Le Service Régional de l'Archéologie, dispose d'une information riche sur les cinq premiers mètres du sous-sol urbain. Cependant cette documentation est dispersée dans de nombreux rapports, dossiers ou articles et sa précision est très variable, les documents couvrant deux siècles d'acquisition. C'est pourquoi ces données ont été réinterprétées à la lumière du référentiel commun à l'archéologie et à la géotechnique. In fine, nous disposons dans la zone d'étude de 53 sondages - au sens géotechnique du mot - utilisables pour l'amélioration du modèle (fig. 1).

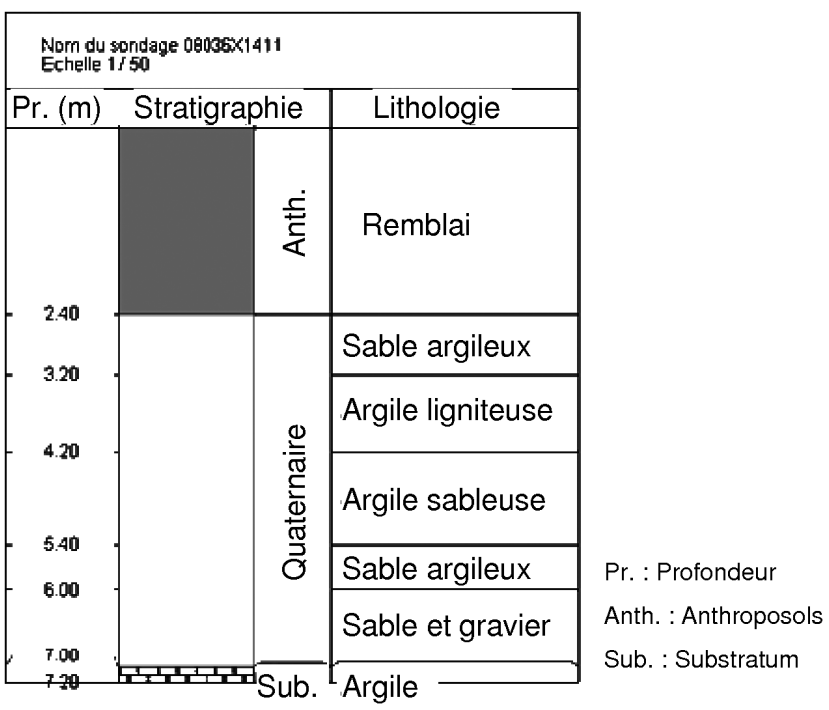

Figure 2 : Exemple d'un log codé.

Figure 2: Encod log example.

L'ensemble des sondages est utilisé pour reconstruire le modelé topographique à la fin du Quaternaire, c'est-à-dire la surface supérieure de la formation quaternaire avant que l'Homme n'occupe ce site. On dispose pour construire le modèle de cette surface de données « vraies » (sondages ayant 
rencontré l'interface recherchée) et de données " d'inégalité » (sondages s'arrêtant avant d'avoir rencontré l'interface recherchée).

\section{Modélisation gÉostatistiQue}

\section{Surfaces modélisées}

Si la première surface que nous souhaitons obtenir est l'interface sol naturel/sol anthropisé, nous nous intéresserons également à modéliser l'interface substratum/Quaternaire, qui permet de mieux comprendre la mise en place du réseau hydrographique dans ce secteur. Le modèle est donc composé d'un empilement de couches, dont les bases (les murs) sont calculées. La surface topographique est élaborée à partir du modèle numérique de terrain du centre-ville. Ce modèle numérique de terrain est basé sur le modèle de l'IGN à pas de $25 \mathrm{~m}$ et résolution verticale de $1 \mathrm{~m}$; cependant, la précision de ce modèle n'étant pas satisfaisante, il a été modifié en utilisant l'ensemble des informations des sondages de la base de données (Marache et al., 2009). L'ensemble du modèle respecte les relations géologiques qui existent entre les trois ensembles stratigraphiques :

- le Quaternaire érode le substratum, en relation avec la mise en place de terrasses fluviatiles (Foucault \& Raoult, 1999);

- l'Anthroposol est également considéré comme une couche érosive.

D'après l'étude géologique du secteur, il semble plus pertinent de reconstruire des surfaces stratigraphiques (correspondant à la situation lors du dépôt) plutôt que des surfaces actuelles (surface initiale incisée ultérieurement par l'érosion). En effet, nous nous trouvons dans un milieu de dépôt qui n’a pas subi de phénomènes tectoniques importants (Autran et Dercourt, 1980), ce qui permet de supposer que les couches, qui constituent le substratum, pourront être sub-horizontales et parallèles entre elles (Lenguin $e t$ al., 1980). Ces surfaces de dépôt initiales ont, par la suite, été creusées lors des différentes phases d'érosion, puis comblées, entre les phases d'érosion, à nouveau par des alluvions (terrasses fluviatiles) (Dubreuilh et al., 1995).

\section{Krigeage sous contraintes d'inégalité}

Le krigeage est une méthode d'interpolation spatiale qui prévoit la valeur d'une variable en des sites non échantillonnés par une combinaison linéaire sans biais et à variance minimale des observations du phénomène en des sites voisins (Matheron, 1962).
Tel que l'indique Cressie (1993), la mise en oeuvre du krigeage s'effectue en suivant certaines étapes qui seront développées dans les paragraphes suivants. La première étape consiste en l'analyse variographique de la variable étudiée. Puis un modèle de variogramme s'ajustant au variogramme expérimental est trouvé et validé par une validation croisée. Cette dernière sous-étape permet de comparer la performance de différents modèles afin de sélectionner celui susceptible de mener aux meilleures prévisions. Finalement, l'interpolation est effectuée par krigeage.

\section{Construction du variogramme expérimental}

En géostatistique, l'idée fondamentale est que la nature n'est pas entièrement "imprévisible». Deux observations proches devraient, en moyenne, se ressembler davantage que deux observations éloignées. C'est pourquoi on cherche préalablement à quantifier cette continuité. La fonction la plus utilisée pour décrire cette continuité est le variogramme (Matheron, 1970).

On estime le variogramme expérimental à l'aide de la fonction $\gamma(\mathrm{h})$ :

$\gamma_{e}(h)=\frac{1}{2 N(h)} \sum_{i=1}^{N(h)}\left[Z\left(x_{i}\right)-Z\left(x_{i}+h\right)\right]^{2}$

où $\mathrm{N}(\mathrm{h})$ est le nombre de paires dont les points sont espacés de $\mathrm{h}, \mathrm{Z}\left(\mathrm{x}_{\mathrm{i}}\right)$ et $\mathrm{Z}\left(\mathrm{x}_{\mathrm{i}}+\mathrm{h}\right)$ les valeurs de la fonction $\mathrm{Z}$ (dans notre cas les altitudes de l'interface) en $x_{i}$ et en $x_{i}+h$.

La fonction $\gamma(\mathrm{h})$ quantifie donc la perte de corrélation moyenne entre deux points quand la distance $h$ entre ces points augmente. Le semi-variogramme atteint (ou non) un plateau. L'atteinte d'un plateau indique qu'à partir d'une certaine distance, il n'y a plus de dépendance spatiale entre les données. Cette distance est nommée portée et le terme palier dénote la valeur du variogramme pour laquelle le plateau se présente. La portée représente la distance au-delà de laquelle deux observations ne sont plus corrélées entre-elles.

Le variogramme expérimental est calculé jusqu’à une distance qui équivaut à la moitié du domaine de l'étude, soit $1 \mathrm{~km}$ dans notre cas. Après avoir vérifié qu'il n'existait pas de directions principales d'anisotropie (c'est-à-dire que la structuration spatiale de la variable est la même quelque soit la direction étudiée), on calcule un variogramme omnidirectionnel (cf. fig. 3 et 4). Pour les deux variables (interfaces sol naturel/sol anthropisé et substratum/Quaternaire), la portée (valeur pour laquelle le variogramme atteint un palier) est d'environ 500 mètres. 


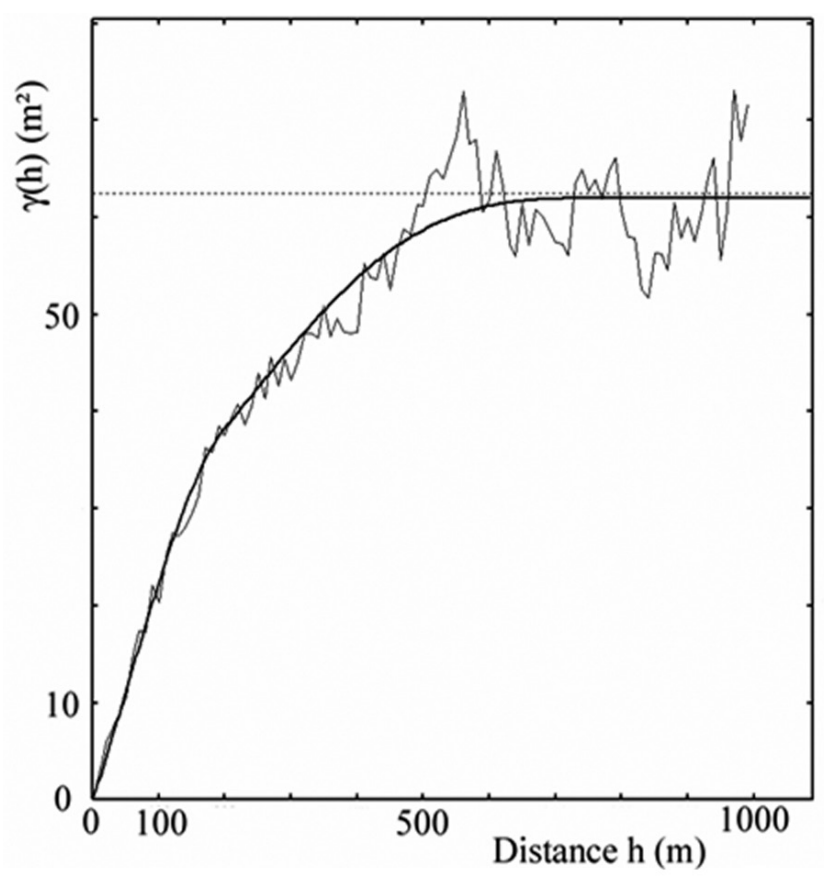

Figure 3 : Variogramme expérimental et modèle de variogramme pour le mur du Quaternaire.

Figure 3: Experimental variogram and variogram model for Quaternary bottom.

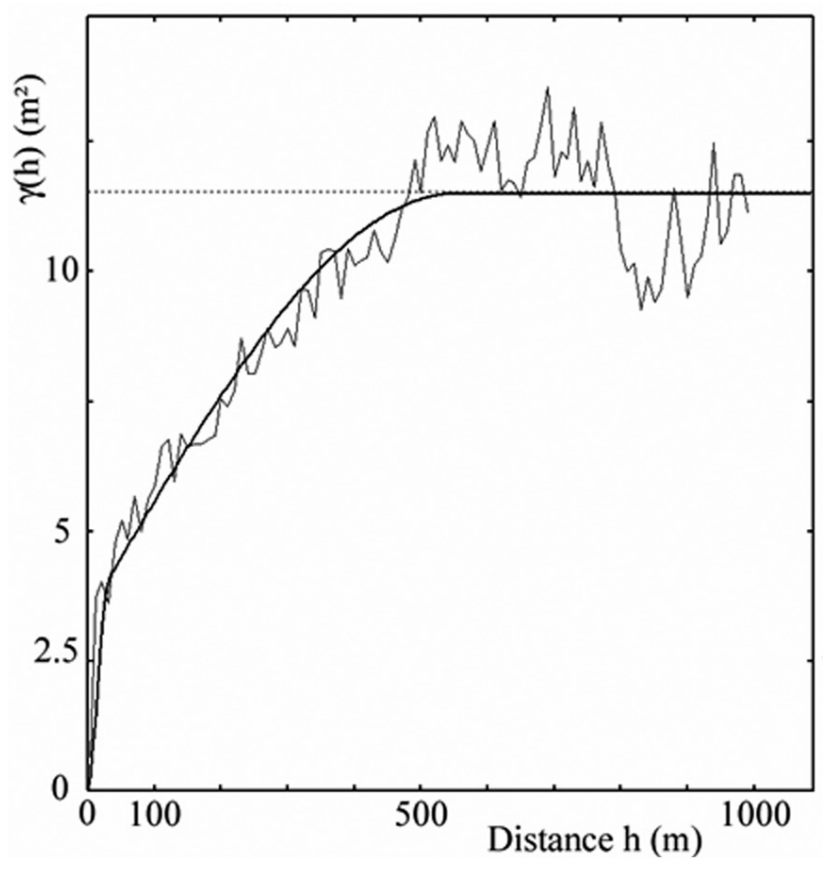

Figure 4 : Variogramme expérimental et modèle de variogramme pour le mur de l'Anthroposol.

Figure 4: Experimental variogram and variogram model for Anthroposoil bottom.

\section{Établissement du modèle de variogramme}

Le variogramme expérimental est insuffisant dès que l'on souhaite aller au-delà de la simple description. Pour répondre au besoin d'estimation et au problème de l'interpolation, on doit disposer d'un modèle de variogramme théorique. Celui-ci ne peut être quelconque (Chilès et Delfiner, 1999) et on utilise alors des fonctions de base (modèles de variogramme sphérique, exponentiel, linéaire...), plusieurs modèles de variogrammes pouvant être additionnés (structures emboîtées).

Le modèle de variogramme quantifie la manière dont les corrélations entre les valeurs d'une variable (ici l'altitude) prise en deux points décroît avec la distance $h$ entre ces deux points.

Après avoir calculé les variogrammes expérimentaux (cf. paragraphe précédent), on identifie des modèles de variogramme (fig. 3 et 4) sous la forme de fonctions qui s'ajustent au mieux aux variogrammes expérimentaux parmi la multitude de modèles existants. Après avoir cherché les modèles s'ajustant au mieux aux variogrammes expérimentaux, on a retenu les deux modèles suivants :

Pour le mur du Quaternaire (fig. 3) :

$-\mathrm{h}<200 \mathrm{~m}$ :

$\gamma(h)=32\left[\frac{3}{2}\left(\frac{h}{200}\right)-\frac{1}{2}\left(\frac{h}{200}\right)^{3}\right]+30\left[7\left(\frac{h}{600}\right)^{2}-\frac{35}{4}\left(\frac{h}{600}\right)^{3}+\frac{7}{2}\left(\frac{h}{600}\right)^{5}-\frac{3}{4}\left(\frac{h}{600}\right)^{7}\right]$

(3)

$-200 \leq \mathrm{h}<600 \mathrm{~m}:$

$\gamma(h)=32+30\left[7\left(\frac{h}{600}\right)^{2}-\frac{35}{4}\left(\frac{h}{600}\right)^{3}+\frac{7}{2}\left(\frac{h}{600}\right)^{5}-\frac{3}{4}\left(\frac{h}{600}\right)^{7}\right]$

$-\mathrm{h} \geq 600 \mathrm{~m}$ :

$\gamma(\mathrm{h})=62 \mathrm{~m}^{2}$

Pour le mur de l'Anthroposol (fig. 4) :

$-\mathrm{h}<100 \mathrm{~m}$ :

$\gamma(h)=4\left[\frac{3}{2}\left(\frac{h}{100}\right)-\frac{1}{2}\left(\frac{h}{100}\right)^{3}\right]+8\left[7\left(\frac{h}{500}\right)^{2}-\frac{35}{4}\left(\frac{h}{500}\right)^{3}+\frac{7}{2}\left(\frac{h}{500}\right)^{5}-\frac{3}{4}\left(\frac{h}{500}\right)^{7}\right](6)$

$-100 \leq \mathrm{h}<500 \mathrm{~m}$ :

$\gamma(h)=4+8\left[7\left(\frac{h}{500}\right)^{2}-\frac{35}{4}\left(\frac{h}{500}\right)^{3}+\frac{7}{2}\left(\frac{h}{500}\right)^{5}-\frac{3}{4}\left(\frac{h}{500}\right)^{7}\right]$

$-\mathrm{h} \geq 500 \mathrm{~m}$ :

$\gamma(\mathrm{h})=12 \mathrm{~m}^{2}$ 


\section{Validation du modèle}

Pour contrôler la qualité du modèle ajusté nous effectuons une validation croisée. Celle-ci consiste à éliminer temporairement un point de l'ensemble des données puis à estimer sa valeur par krigeage à l'aide des données voisines et du modèle de variogramme qui a été ajusté (Isaaks \& Srivastava, 1989; Arnaud \& Emery, 2000; Cressie, 1993; Wackernagel, 2003). Cette opération est répétée pour tous les points. En tout point d'échantillonnage nous obtenons donc une altitude estimée $\mathrm{z}^{*}$, accompagnée de son écart-type de krigeage. Cet indicateur quantifie la dispersion autour de la valeur vraie (connue dans le cas d'une validation croisée) autour de la valeur estimée. L'estimation peut alors être comparée avec l'altitude réelle $\mathrm{z}$.

On choisit d'utiliser pour la validation de la reconstruction d'un point uniquement les points situés dans un voisinage circulaire de $500 \mathrm{~m}$, appelé voisinage de krigeage (distance jusqu'à laquelle on va chercher de l'information pour estimer la valeur d'un point), cette distance étant au moins égale à la portée. Les résultats de cette validation nous permettent d'accepter les modèles choisis pour la reconstruction.

\section{Définition des données d'inégalité}

Le krigeage sous contraintes d'inégalités est une technique qui permet d'utiliser une variable qui n'est pas définie par une valeur exacte en un point, mais par un intervalle dont les bornes constituent les données d'inégalité (Langlais, 1990). Dans notre cas, les données "vraies " sont représentées par les valeurs exactes : par exemple, pour l'interface substratum/ Quaternaire, la variable " mur exact du Quaternaire» est reconnue lorsqu'on rencontre le Quaternaire au contact du substratum. Les données d'inégalités sont représentées par les valeurs seuils que sont les variables " mur inférieur " et " mur supérieur "; dans ces deux expressions, le terme de " mur » n'est pas à prendre au sens géologique strict mais signifie que l'on a des bornes inférieure et supérieure encadrant le mur exact.

Par exemple, la formation Quaternaire repose sur le substratum par le biais d'une surface d'érosion. A partir de cette configuration d'origine, on peut envisager trois cas :

- dans le premier (A), le sondage n'atteint pas le substratum. On sait alors que la transition Quaternaire/substratum est sous la base du forage, le sondage fournit alors une borne supérieure de cette interface,

- dans le second cas (B), on identifie des anthroposols qui reposent sur le Quaternaire. Plus bas, la transition Quaternaire/substratum est reconnue; c'est donc une valeur de mur exact,
- dans le troisième cas (C), l'anthroposol est directement au contact du substratum. Cela correspond à l'érosion complète du Quaternaire (cas C), voire à l'érosion supplémentaire d'une partie du substratum (cas Cbis). On connait alors la cote minimale qu'a pu avoir la base du Quaternaire au cours des temps (borne inférieure du mur du Quaternaire).

Le krigeage sous contraintes d'inégalités est conduit en deux étapes :

- la première étape consiste à simuler, au droit du point où l'on possède une donnée d'inégalité, une valeur en accord avec les données d'inégalité (fig. 5) et le modèle de variogramme défini; on dispose ainsi d'un ensemble de données complémentaires qui permettent d'affiner le modèle. Il est à noter que pour cette étape, les données de départ doivent satisfaire une distribution gaussienne; si tel n'est pas le cas, une anamorphose est nécessaire.

- la seconde étape est un krigeage " traditionnel " qui utilise les données vraies et les données simulées à l'étape précédente pour construire la surface recherchée.

\section{Reconstruction, par krigeage sous contraintes} d'inégalités, des surfaces et estimation de la qualité de cette reconstruction

La reconstruction fournit en tout point $(\mathrm{x}, \mathrm{y})$, selon une grille à mailles carrées de $25 \mathrm{~m}$, une estimation de l'altitude $\mathrm{z}^{*}$ et un écart-type de krigeage associé, que l'on peut exprimer sous la forme d'un $\Delta z$. Cette valeur, cartographiée sur l'ensemble du domaine, permet d'apprécier l'incertitude que l'on a sur le positionnement (ici l'altitude) des interfaces. Elle est utile, soit pour une analyse directe d'incertitude, soit pour guider des reconnaissances futures, en privilégiant

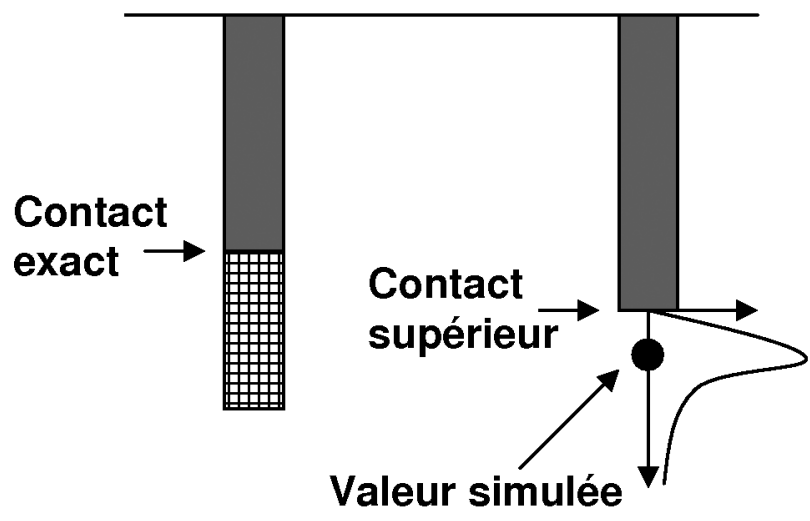

Figure 5 : Simulation de valeur pour une donnée d'inégalité. Figure 5: Simulation of a value for an inequality data. 
si besoin les zones de forte incertitude pour y obtenir des données complémentaires.

On reconstruit tout d'abord la surface du mur du Quaternaire (fig. 6). La visualisation 3D de cette surface met en évidence l'incision du réseau hydrographique dans le substratum.

Ensuite on reconstruit celle du mur de l'Anthroposol (fig. 7). Ces cartes sont une étape intermédiaire avant «l'assemblage » du modèle.

\section{a) Cartographie de l'altitude}

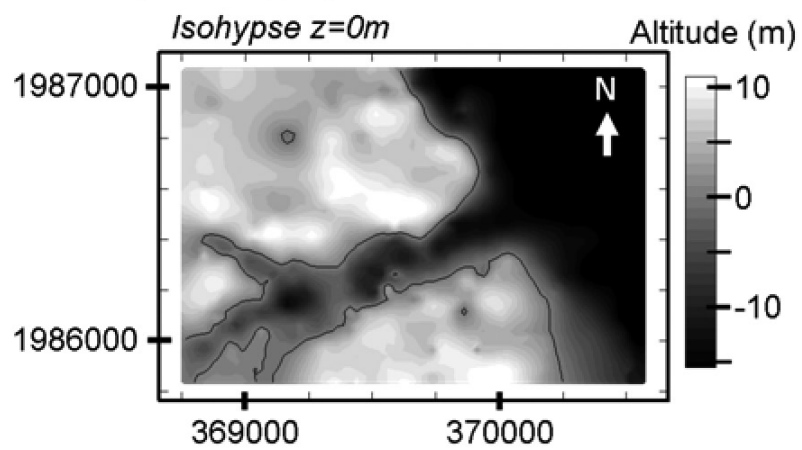

b) Vue tridimensionnelle de la surface Échelle verticale $x 10$

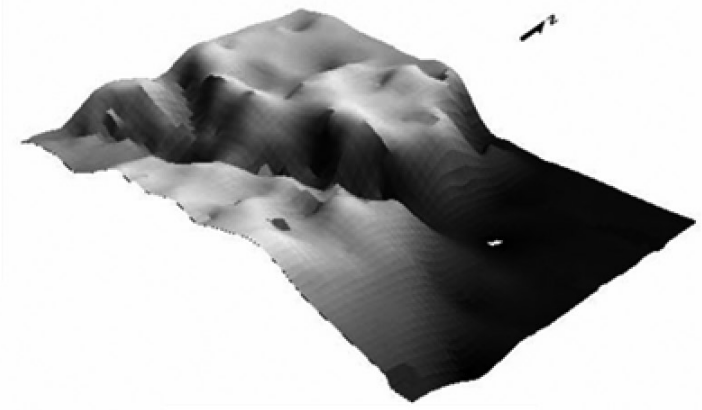

c) Cartographie de l'écart-type de krigeage

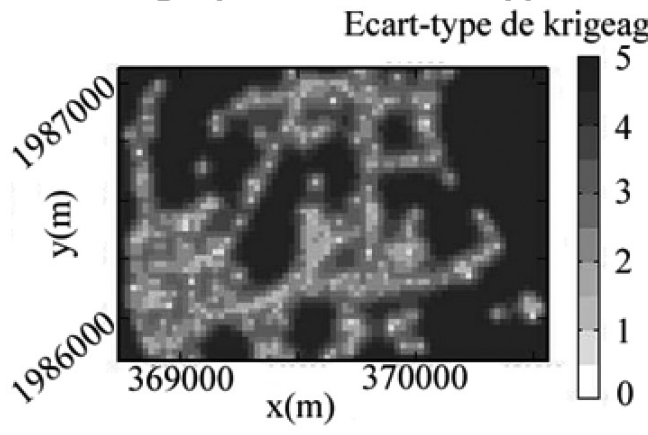

Figure 6: Reconstruction du mur du Quaternaire.

Figure 6: Rebuilding of Quaternary bottom.

\section{a)Cartographie de l'altitude}

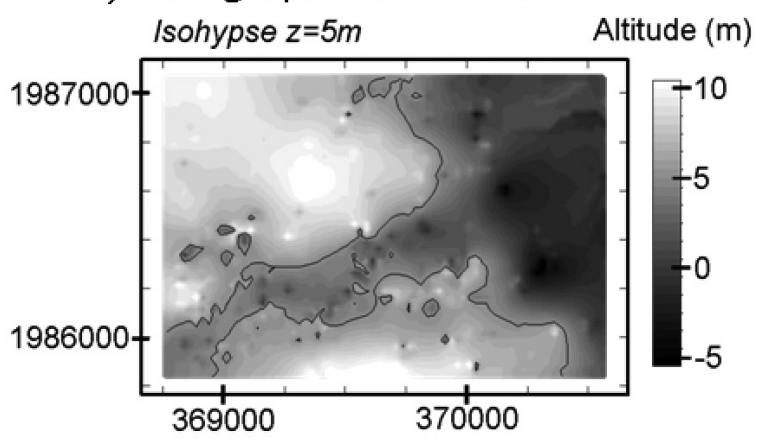

\section{b) Vue tridimensionnelle de la surface} Échelle verticale x10

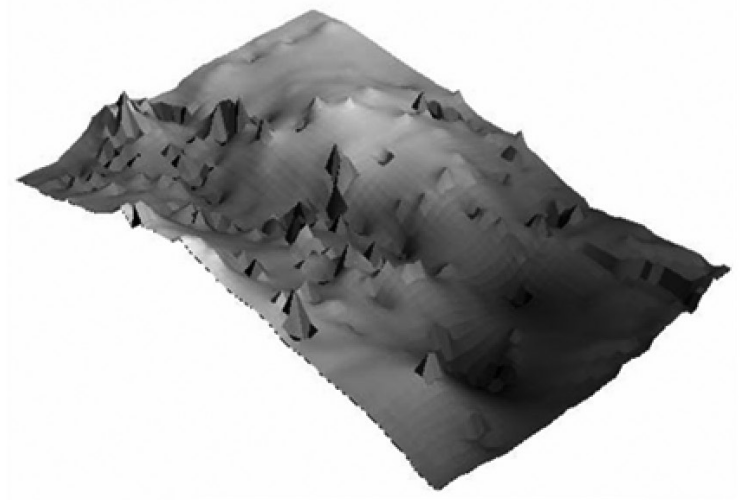

c) Cartographie de l'écart-type de krigeage

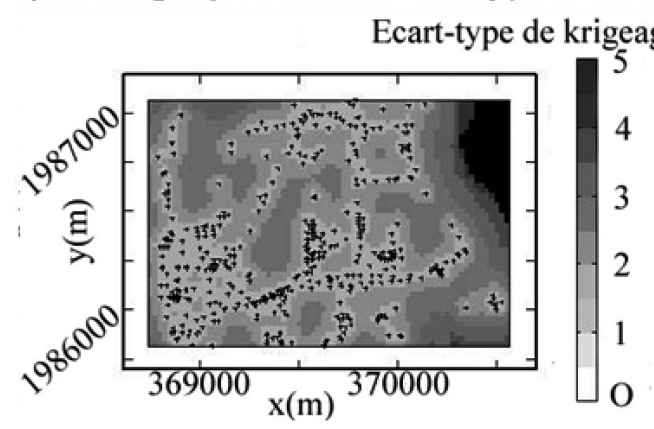

Figure 7 : Reconstruction du mur de l'Anthroposol. Figure 7: Rebuilding of Anthroposoil bottom.

\section{RÉsultats}

Après avoir interpolé et validé les surfaces, on recompose le modèle par assemblage (les surfaces sont intersectées suivant la logique de dépôt/érosion tout en respectant les contraintes topographiques) afin de ne conserver les surfaces qu'aux endroits où elles existent aujourd'hui. En effet les surfaces reconstruites par le modèle sont celles qui existaient 
à la fin de leur séquence de dépôt et non pas celles que l'on rencontre actuellement.

Afin de suivre les commentaires des résultats et leur analyse, une carte simplifiée du centre-ville de Bordeaux est présentée en fig. 8 .

\section{Cartes}

La surface la plus ancienne du modèle est la base des formations quaternaires (fig. 9). Les zones en blanc sont celles qui ont été recoupées par la surface de l'Anthroposol ou la topographie (plus récente), c'est-à-dire celles où l'Anthroposol se retrouve directement au contact du substratum ou celles où le substratum est affleurant. On a donc dans ces zones, soit une absence, soit une érosion totale des dépôts quaternaires.

La vallée de la Devèze ainsi que celle du Caudéran s'inscrivent profondément dans le substratum (fig. 9), par contre le Peugue semble absent sur ce secteur, ce qui fait penser à une confluence avec la Devèze plus à l'ouest de ce secteur d'étude. On constate que la paléo-Garonne avait un lit beaucoup plus large que la Garonne d'aujourd'hui, et que ce lit s'étendait plus largement vers l'ouest, sous la place des Quinconces. La Devèze était alors un affluent majeur de cette paléo-Garonne.
La base de l'Anthroposol (fig. 10) est la première surface que l'on rencontre dans ce modèle en dessous de la surface topographique. Les zones en blanc sont celles où l'épaisseur d'Anthroposol dans le modèle est nulle, c'est-à-dire celles où l'on rencontre directement des terrains «naturels » à l'affleurement. Sur cette carte on note que le fond du lit du Peugue (qui ne se marque que très légèrement) se situe à une altitude plus élevée que celle du lit de la Devèze (niveau de gris plus foncé).

\section{Coupes}

On a choisi de tracer une première coupe dans le modèle le long de la rue Sainte-Catherine (cf. localisation fig. 8 et coupe fig. 11). Une comparaison peut en être faite avec une coupe qui avait été tracée à partir des documents produits lors de l'avant-projet du métro à Bordeaux dans les années 1980 (coupe S-N entre le cours Victor-Hugo et la place des Quinconces; Barraud et al., 1988). Les sondages utilisés pour la coupe du métro font partie de ceux utilisés pour le modèle. Les différences entre les deux coupes proviennent du caractère tridimensionnel des surfaces reconstruites dans le modèle, et de la prise en compte dans ce modèle des informations existant hors du plan de coupe. Les échelles

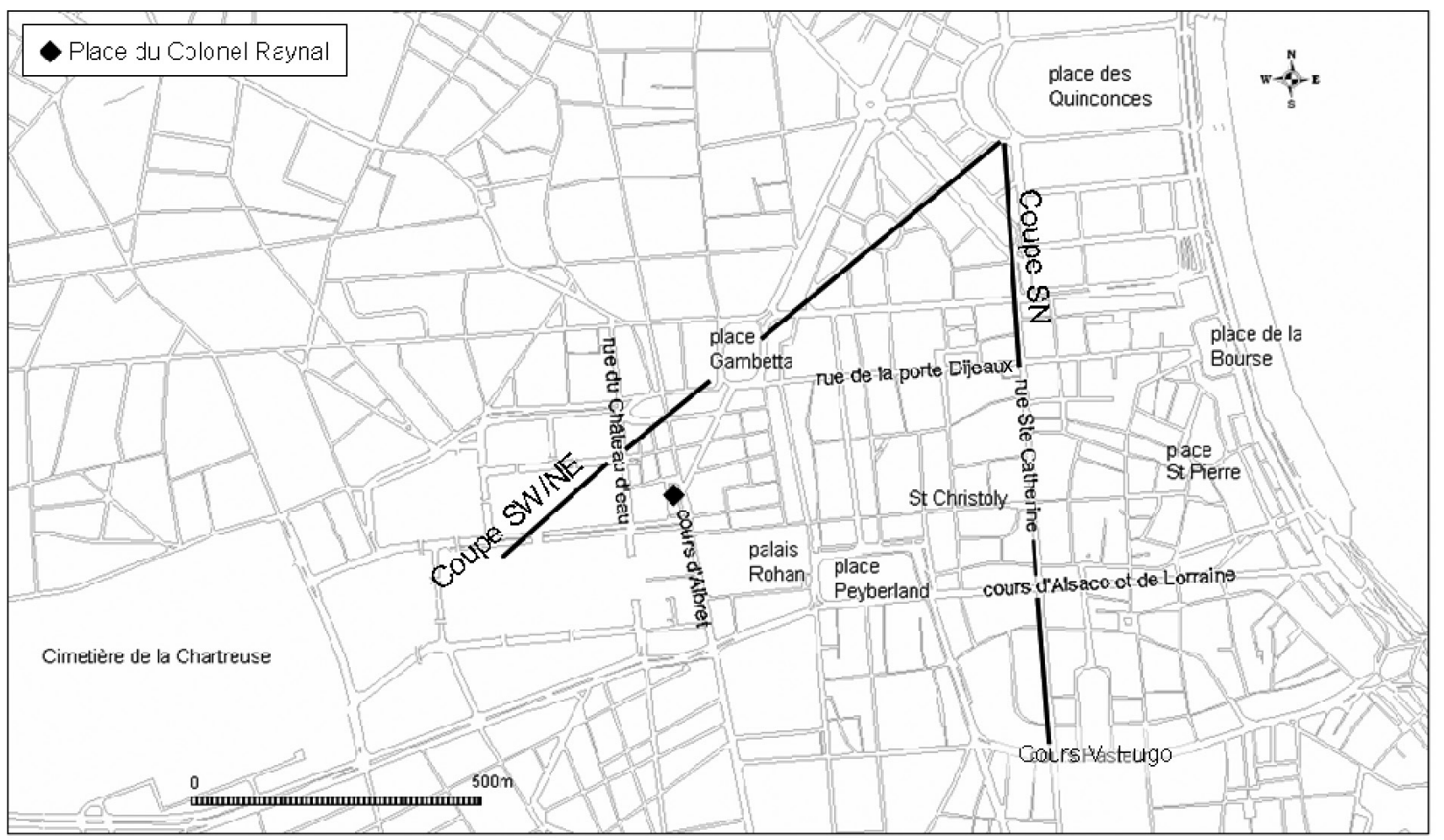

Figure 8 : Plan simplifié de Bordeaux.

Figure 8: Bordeaux simplified map. 


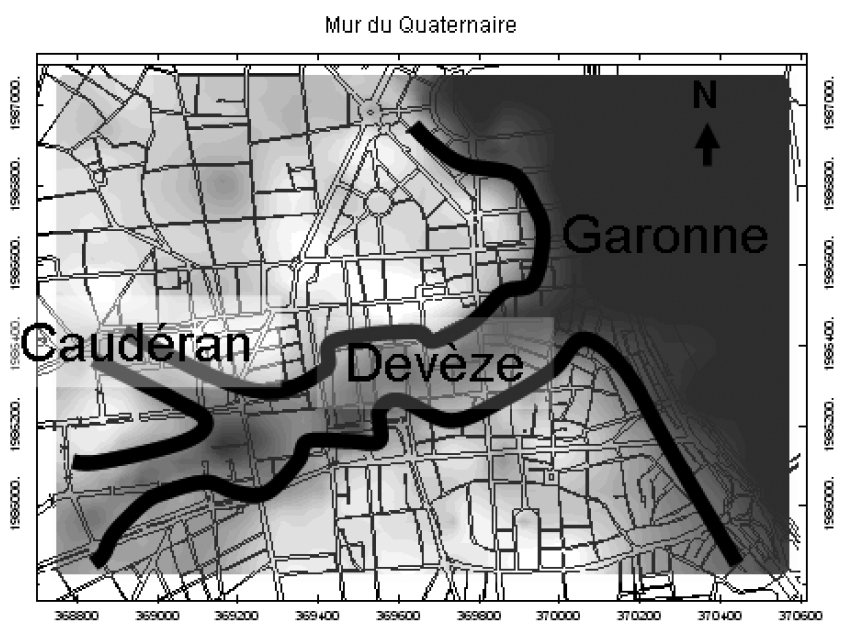

Figure 9: Analyse du mur du Quaternaire. Figure 9: Quaternary bottom analysis.

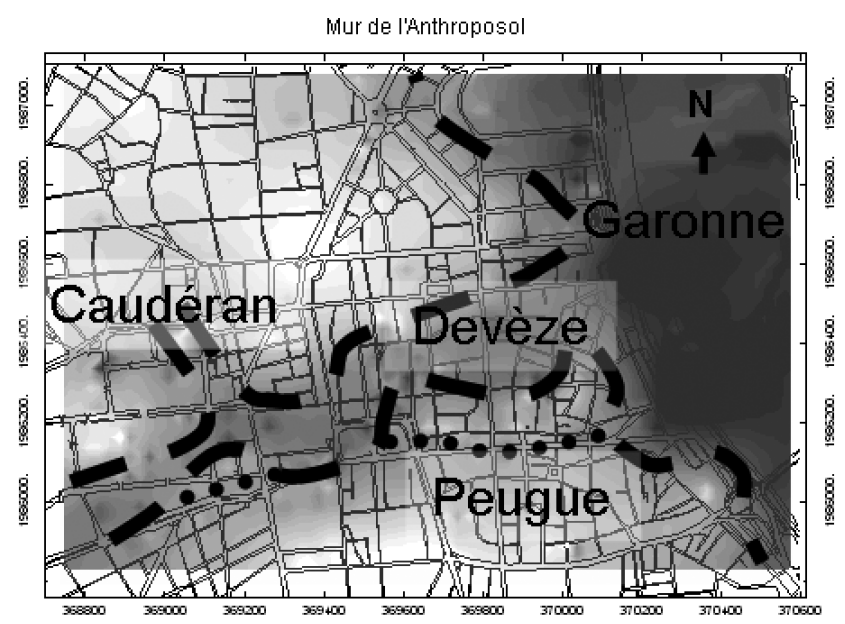

Figure 10 : Analyse du mur de l'Anthroposol.

Figure 10: Anthroposoil bottom analysis.
Coupe S-N dans le modèle

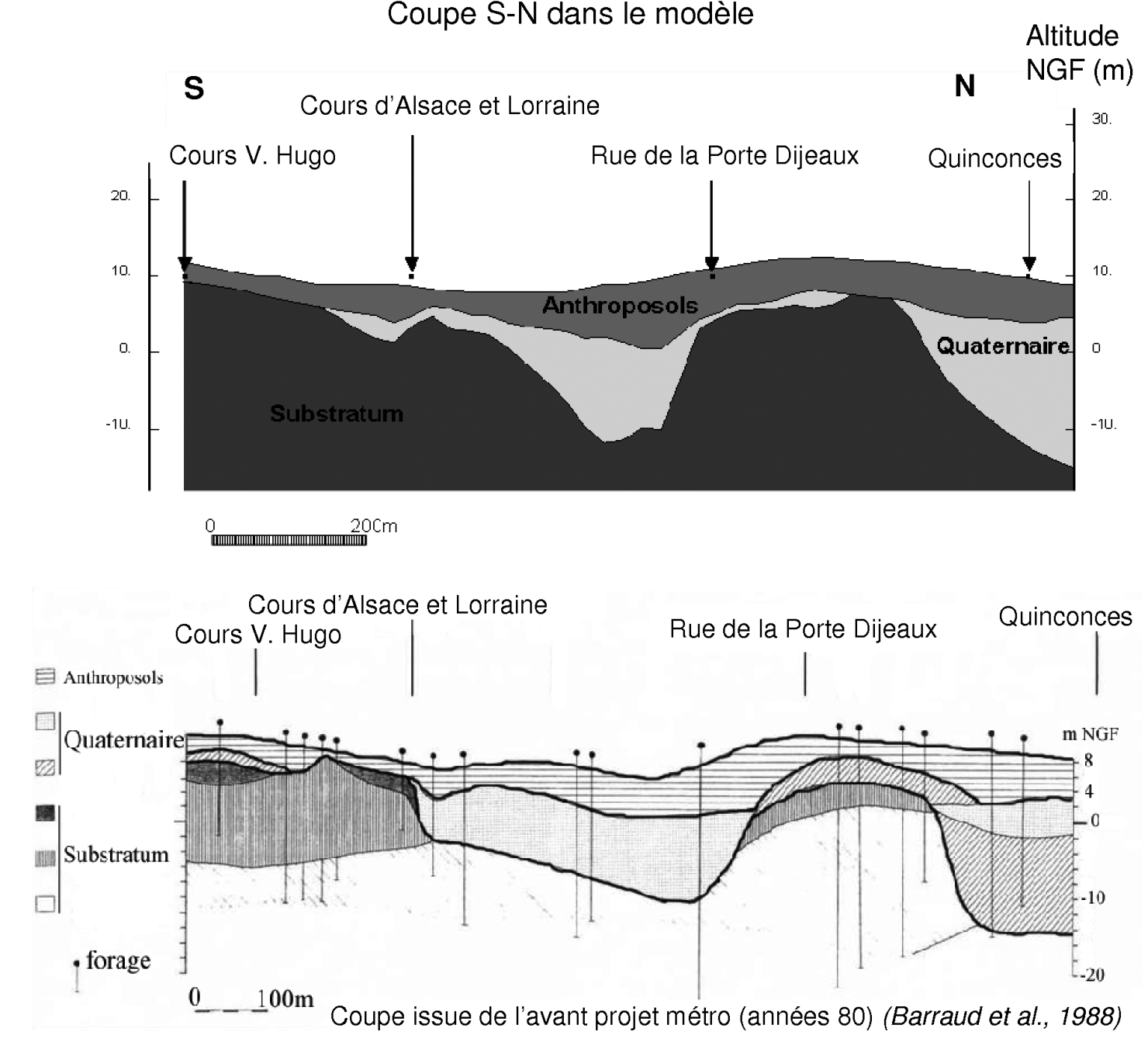

Cours d'Alsace et Lorraine

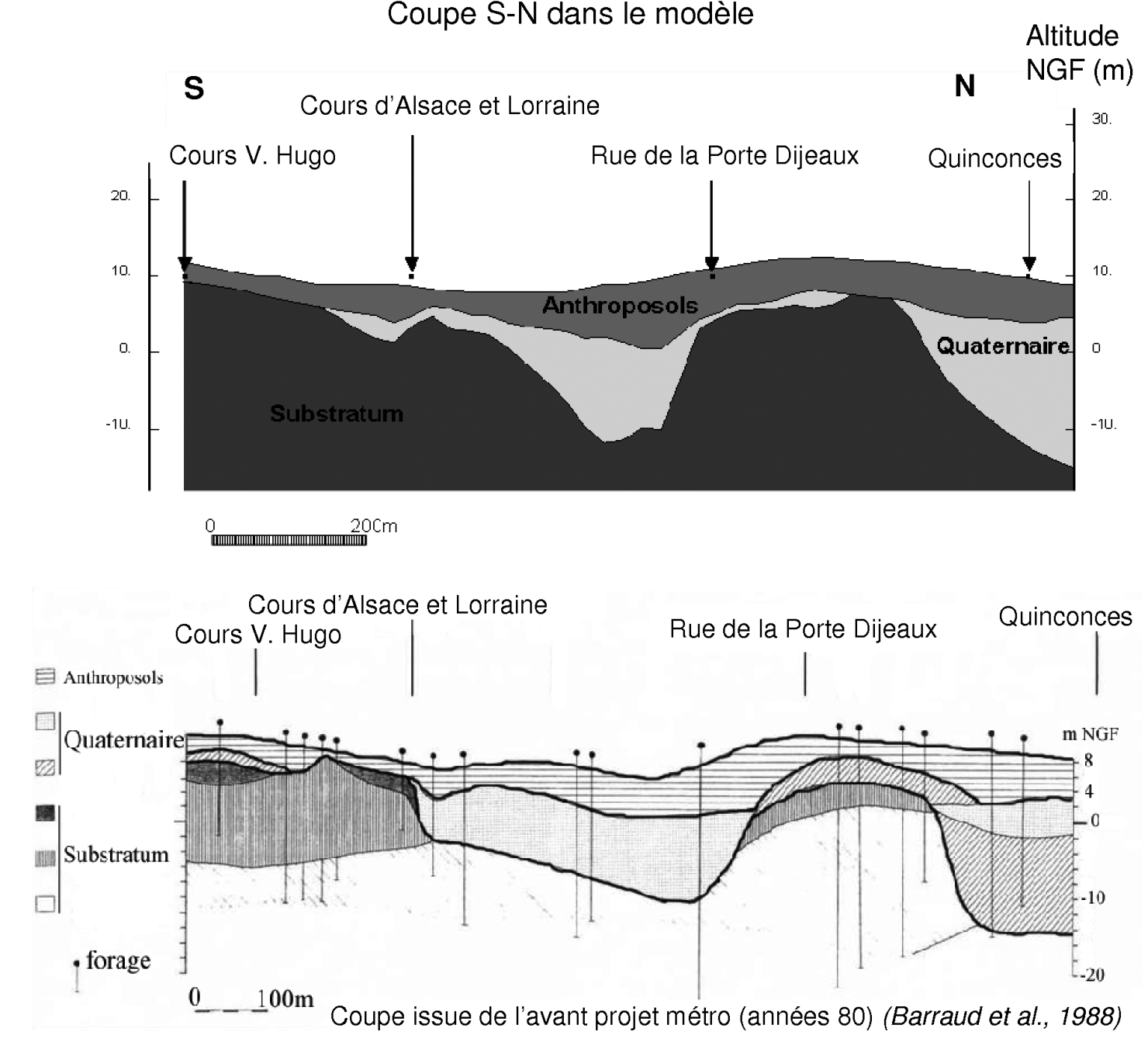

Figure 11:

Coupe S-N dans le modèle et le long de la ligne $\mathrm{A}$ du métro.

Figure 11: $S-N$

cross-section in the model and along the A-line subway.

ArcheoSciences, revue d'archéométrie, 34, 2010, p. 25-37 
verticales et horizontales sont respectivement les mêmes sur les deux coupes (l'échelle verticale est par contre dilatée par rapport à l'échelle horizontale).

Le niveau de détail du modèle est différent de celui de la coupe. Il s'agit en effet d'une coupe lithologique alors que le modèle que nous avons établi est un modèle stratigraphique. Néanmoins on peut traduire la coupe de l'avantprojet métro en termes de stratigraphie et ainsi comparer la base des Anthroposols et la base du Quaternaire entre les deux coupes.

On remarque immédiatement que les deux coupes sont très similaires sur la moitié nord. On retrouve le creusement de la paléo-Garonne sous les Quinconces, ainsi que l'incision de la vallée de la Devèze dans le substratum au sud de l'intersection des rues Sainte-Catherine et Porte-Dijeaux. Les flancs de cette vallée sont très abrupts, et deux platesformes de substratum sont isolées de part et d'autre de la vallée.

Cependant on note un décalage sur la moitié sud, car si on observe des modelés similaires (petite remontée du substratum qui sépare la vallée de la Devèze de celle du Peugue), ils sont décalés. La carte des cours d'eau au XviII ${ }^{\mathrm{e}}$ siècle indique que le Peugue coulait au sud du cours d'Alsace-etLorraine, il est donc probable qu'il y a eu une erreur sur la coupe tirée des documents du métro. En effet, sur la coupe originelle que nous avons consultée, les surfaces sont tracées en pointillés, l'auteur ayant émis des réserves quant à la localisation de ces interfaces. Ces pointillés ont disparu dans une version ultérieure de la coupe. Cette coupe permet également de visualiser que le fond du lit du Peugue se situe à une altitude plus élevée que celle du lit de la Devèze, comme déjà mentionné dans l'analyse de la figure 10. On peut également noter à ce même endroit une très légère entaille du substratum qui n'était pas visible sur la carte de la figure 9. Cependant une différence de plus dix mètres entre les altitudes des deux fonds de rivières (Peugue et Devèze qui appartiennent au même système, ne sont distants que de $300 \mathrm{~m}$ et se jettent dans la Garonne moins de $500 \mathrm{~m}$ plus loin) n'est pas anodine et ne peut pas vraiment se justifier autrement que par une différence de chronologie d'ouverture comme cela sera expliqué dans la partie suivante. Cette entaille du substratum, qui n'est certainement que partiellement naturelle (surcreusements réalisés à la fin du $\mathrm{XVIII}^{\mathrm{e}}$ siècle), est donc postérieure à celle faite par la Devèze

On a procédé à une seconde coupe SW/NE dans le modèle afin de la comparer à la deuxième coupe tracée dans le cadre de l'avant-projet du métro (cf. fig. 12). De même que précédemment, les échelles verticales et horizontales sont respectivement les mêmes sur les deux coupes (l'échelle verticale est par contre dilatée par rapport à l'échelle horizontale).
À nouveau on retrouve deux coupes très proches dans leur modelé, avec sur la droite le lit de la paléo-Garonne, et sur la gauche celui du Caudéran.

\section{Proposition d'interprétation}

Dans ce modèle, il apparaît clairement que le Peugue, qui n'est pas visible sur la carte du mur du Quaternaire (fig. 9), n'intervient que tardivement dans le paysage bordelais, et que la phase de non convergence avec la Devèze, envisagée dans l'hypothèse préalable, n'a aucune raison d'être. On peut même constater que le niveau de base de la saignée correspond sensiblement à des occupations archéologiques du Haut Moyen Âge (Debord et Gauthier, 1982); ce qui, dans la mesure où il s'agit du même système fluvial, tend à induire une synchronie.

Il faut donc envisager une nouvelle hydrographie originelle : le Peugue, arrivant de Pessac (au Sud-Ouest) et la Devèze, qui vient de Mérignac (à l'Ouest) - jusqu'ici c'est le système actuel - confluent quelque part au sud du cimetière de la Chartreuse ou un peu en aval; ils reçoivent le Caudéran entre la rue du Château-d'eau et la place du Colonel-Raynal et traversent Bordeaux selon le même tracé que la Devèze médiévale.

Dans cette hydrographie (fig. 13), on peut distinguer deux systèmes : un système fluviatile, sensiblement jusqu'au cours d'Albret; un système intertidal en aval, l'estey proprement dit, une lagune remplie par les marées hautes et drainée à marée basse par la rivière. C'est ce système que va exploiter le port romain (Régaldo-Saint Blancard, 2005).

Les modèles de la base de l'Anthroposol et du Quaternaire sont conformes à cette description. Le système intertidal y est marqué par de forts élargissements du lit, tandis que le système fluviatile correspond plutôt à des saignées étroites. On pouvait, lors de la réalisation du projet Riviera, se poser la question de savoir si le régime intertidal ne dépassait pas à une époque la confluence du Caudéran; c'est maintenant chose pratiquement démontrée par les installations portuaires retrouvées dans une fouille menée en 2009 sur le cours du maréchal Juin, encore inédite. Les élargissements du système intertidal sont susceptibles de variations latérales entre les modèles de la base de l'Anthroposol et du Quaternaire. Ils sont entrecoupés par des étranglements et l'on peut y voir deux causes : soit des étalements et des rétrécissements localisés du lit en fonction des terrains rencontrés, soit la division et le regroupement de deux ou plusieurs chenaux autour d'îlots. Il semble impératif que ces configurations aient été assez durables pour marquer le relief et des carottages pourraient donner une indication dans un sens ou dans l'autre. 


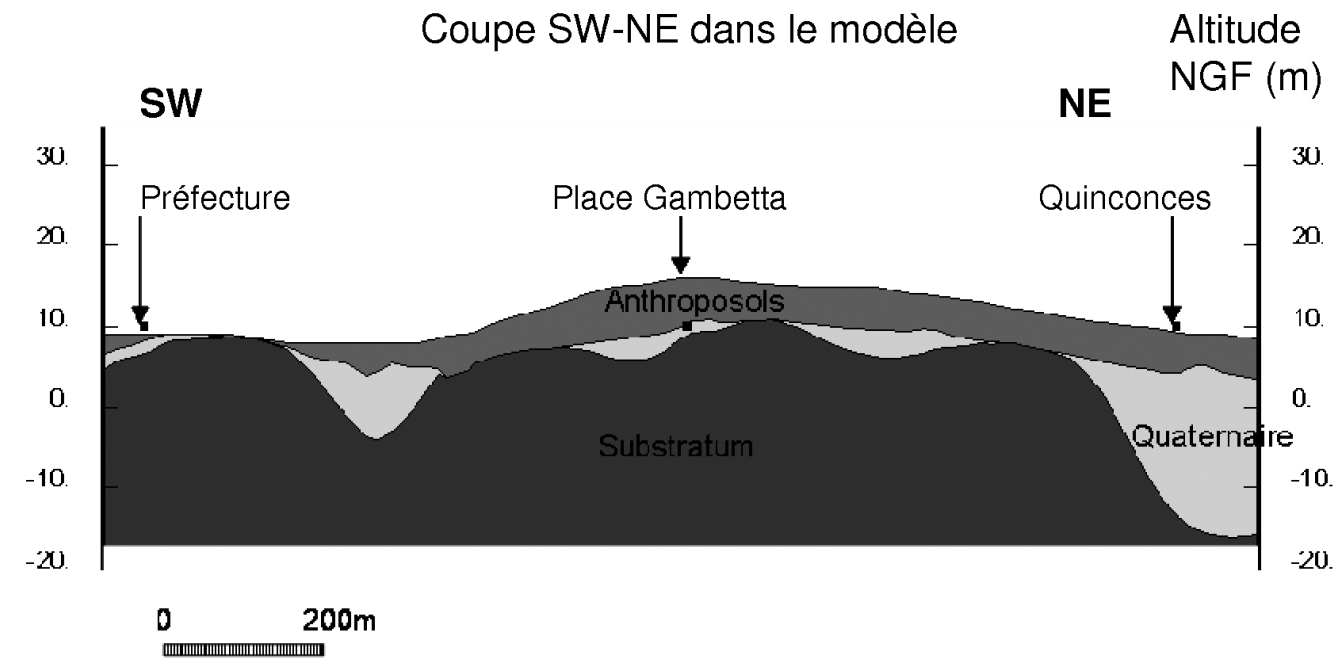

Figure 12 : Coupe SW-NE dans le modèle et le long de la ligne B du métro.

Figure 12: $S W-N E$ cross-section in the model and along the B-line subway.

Place Gambetta

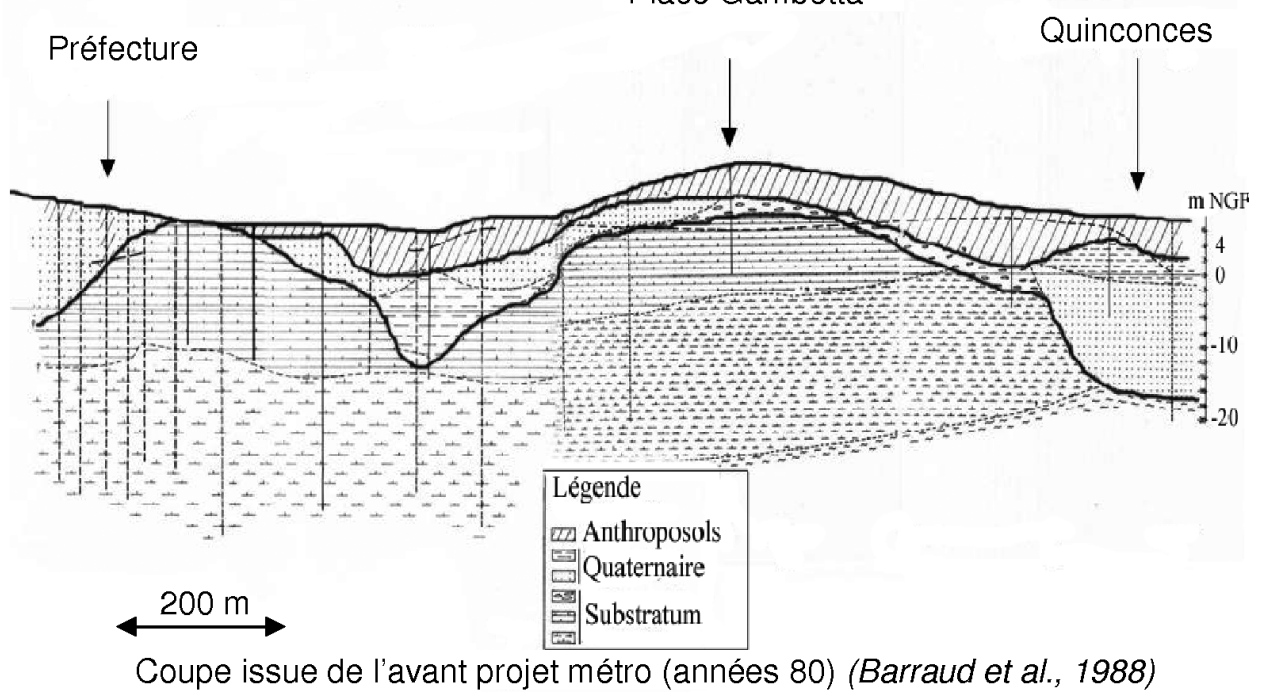

Les fouilles de Saint-Christoly ont observé la confluence de deux chenaux durant le haut empire ( $\mathrm{I}^{\mathrm{er}}$ au $\mathrm{III}^{\mathrm{e}}$ siècle), disparaissant vers 300 , soit lors de la construction de l'enceinte (Lavaud [dir.], 2009). Le bras arrivant de l'ouest et repartant vers l'est fut assimilé à la Devèze; celui arrivant du sud-ouest au Peugue (Debord et Gauthier, 1982). Plus récemment, on a préféré un système de plusieurs bras, naturels ou artificiels. Cette interprétation semble bien coïncider avec l'ensemble des observations archéologiques récentes (cf. ci-dessus).

Dans une perspective analogue, en réinterprétant des données archéologiques anciennes sur le port antique de Bordeaux et en s'appuyant sur des archives jusqu'alors non exploitées, on a pu démontrer (Régaldo-Saint Blancard, 2005) que l'embouchure de la Devèze, auprès de l'église
Saint-Pierre, se divisait en deux bras autour d'un îlot, sans doute dès le début de notre ère; que, vers 300 , on ferma celui du sud par un quai maçonné d'une dizaine de mètres de large et par l'enceinte, elle-même large de 4,50 m; que le bras nord passait à l'arrière de notre place de la Bourse où les récentes fouilles l'ont observé (Gerber [dir.], 2006).

Partant de ces observations, on privilégierait plutôt un réseau de bras enserrant un chapelet d'îlots. On aurait un cas analogue sur ces mêmes rivières, avec l'île qui marquait le port médiéval non loin du marché, et d'autres exemples sur l'Eau-Bourde au sud de Bordeaux, voire sur la Cantinolle, au nord de la Communauté Urbaine. Un tel réseau interviendrait dans un contexte sédimentaire très humide, ce que semble pouvoir provoquer la confrontation des systèmes 


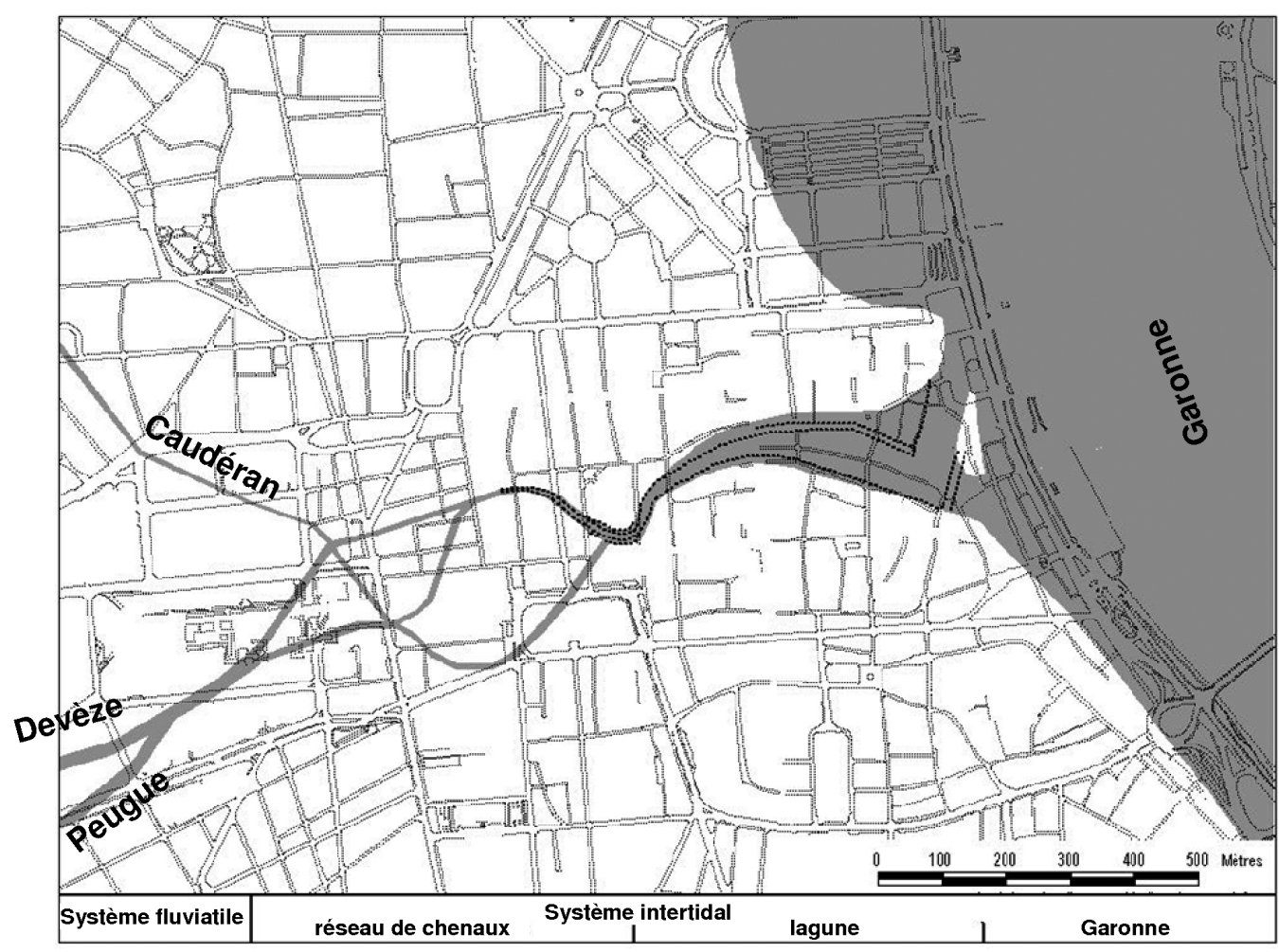

Figure 13 : Proposition d'interprétation : le réseau hydrographique de Bordeaux au début de l'époque romaine.

Figure 13: Proposition of interpretation: Bordeaux's hydrographic network at the beginning of the Roman age. fluviatile et intertidal. Ces îlots étant logiquement alluvionnaires, si les chenaux étaient susceptibles de varier, géologiquement ils apparaîtraient comme des étalements. Toutefois, il semble qu'il convient, aussi bien pour tenir compte des constats géologiques que des observations et interprétations archéologiques, d'ouvrir aussi largement que possible la lagune en aval. D'où la proposition de diviser le système intertidal en deux parts : un réseau en tresse et une lagune. Quant à la confluence ancienne des deux rivières, il est plausible qu'elle soit représentée par l'élargissement sud-ouest du modèle, qui semble un peu trop éloigné de la Garonne pour être soumis à l'influence des marées. Dans le cadre de cette interprétation, la ville romaine contiendrait progressivement ces bras, notamment vers 70 où l'on a pu constater une pression sur le bras aboutissant à Saint-Christoly, et vers 300 (cf. ci-dessus). Le Peugue interviendrait donc tard. On peut retenir la même logique proposée initialement : un envasement de la Devèze en amont de sa partie intra muros, attestée par les fouilles de Saint-Christoly, le frayement d'un débouché au sud de la ville (l'enceinte antique fait barrage et elle est adossée au plateau nord); ce phénomène devant intervenir avant le $\mathrm{XI}^{\mathrm{e}}$ siècle (attestations du marché et du Peugue à partir de ce siècle, vers 1050/1070).

\section{Conclusion}

La réalisation d'un modèle géométrique tridimensionnel sur ce secteur du centre-ville de Bordeaux en utilisant principalement les données lithologiques des forages géotechniques a permis de voir sous un jour nouveau l'évolution des cours d'eau du centre historique et de montrer que l'image usuelle qu'avaient les archéologues sur l'hydrographie de ce secteur était incomplète. Il est donc important de souligner que des données, dont l'échelle de lecture semblait grossière comparée à celle utilisée par les archéologues, se révèlent capitales pour la construction d'une image d'ensemble. Le modèle fournit aussi une estimation du potentiel archéologique, en cartographiant l'épaisseur de l'Anthroposol dans le secteur du centre ville. Dans les deux cas, l'utilisation des méthodes géostatistiques pour la construction du modèle permet d'attacher une précision/incertitude aux estimations de cotes et d'épaisseur. 


\section{Remerciements}

Ce travail a été conduit dans le cadre du Projet RIVIERA (RIsque en VIlle : Equipements, Réseaux et Archéologie), soutenu par le Réseau Génie Civil et Urbain. Les auteurs tiennent à remercier l'ensemble des partenaires du projet (brgm Orléans, DRAC Aquitaine, Lyonnaise des Eaux, Ville de Pessac) ainsi que la Communauté Urbaine de Bordeaux, pour la mise à disposition des données.

\section{Bibliographie}

Arnaud, M. et Emery, X., 2000. - Estimation et interpolation spatiale, Hermes Science Publications, Paris.

Autran, A. et Dercourt, J., 1980. - Les grands bassins sédimentaires mésozoïques et cénozoïques, in Évolutions géologiques de la France, Mémoire du BRGM, n 107.

Barraud, D., Berthault, F., Caillat, P., Nony, D., Sireix, C. et Tilhard, J.-L., 1988. - Le site de "La France ", origines et évolution de Bordeaux antique, Aquitania, 6, p. 1-59.

Barraud, D., Linères, J. et Maurin, L.,1996. - Bordeaux, in L. Maurin et al. (dir.), Enceintes gallo-romaines d'Aquitaine. DAF, Paris, t. 53, p. 15-80.

Chilès, J.-P. et Delfiner, P., 1999. - Geostatistics: Modelling spatial uncertainty, Wiley Interscience.

Cressie, N., 1993. - Statistics for spatial data, Wiley Interscience.

Debord, P. et Gauthier, M., 1982. - Bordeaux Saint-Christoly, sauvetage archéologique et histoire urbaine, Catalogue d'exposition, Musée d'Aquitaine et Direction régionale des Antiquités historiques d'Aquitaine.

Dubreuilh, J., Capdeville, J.-P., Farajanel, G., Karnay, G., Platel, J.-P. et Simon-Coinçon, R., 1995. - Dynamique d'un comblement continental néogène et quaternaire : l'exemple du bassin d'Aquitaine, Géologie de la France, 4, p. 3-26.

Etienne, R., 1962. - Histoire de Bordeaux - Tome I : Bordeaux antique, Fédération Historique du Sud-Ouest.

Foucault, A., Raoult, J.-F., 1999. - Dictionnaire de géologie. Masson, $4^{\mathrm{e}}$ édition.

Galinié, H., Laurent, A., Rodier, X., Breysse, D., Houy, L., Niandou, H. et Breul, P., 2003. - Utilisation du pénétromètre dynamique de type PANDA en milieu urbain pour l'évaluation et la caractérisation du dépôt archéologique. Archeosciences, 27, p. 15-26.

Gerber, F. (dir.), 2006. - Des rivages garonnais de Burdigala au port de la Mer : étude de 2000 ans de rapports entre Bordeaux et son fleuve. Rapport final de fouilles préventives, Bordeaux chantiers Bourse, Saint-Rémi, Jean-Jaurès et Gabriel, janvier 2002 à septembre 2003, 12 vol., Pessac, INRAP, Bordeaux, SRA.
IsaAKs, E. et SrivastaVa, R., 1989. - Applied Geostatistics, Oxford University Press, New York.

Jean-Courret, E., 2006. - La morphogénèse de Bordeaux, des origines à la fin du Moyen Âge, Thèse de doctorat, Université Bordeaux 3, France.

LANGLAIS, V., 1990. - Estimation sous contraintes d'inégalités, Thèse de doctorat, Ecole Nationale Supérieure des Mines de Paris, France.

Lavaud, S. (dir.), 2009. - Atlas historique des villes de France, Bordeaux, Bordeaux, Ausonius. 3 tomes.

Lenguin, M., Mouline, M., Sellier, E., Capdeville, J.-P. et Dubreuihl, J., 1980. - Tertiaire bordelais, in Le bassin d'Aquitaine et sa marge pyrénéenne, 26 Congrès Géologique International, Bull. Cent. Rech. Explor. - Prod. Elf-Aquiatine, Mém. 3, p. 79-116.

Marache, A., Breysse, D., Piette, C. et Thierry, P., 2009. Geological and geotechnical modelling at the city scale using statistical and geostatistical tools: The Pessac case (France), Engineering Geology, 107(3-4), p. 67-76.

Matheron, G., 1962. - Traité de géostatistique appliquée, Tome I. Mémoires du BRGM n 14, Editions Technip, Paris.

Matheron, G., 1970. - La théorie des variables régionalisées et ses applications, Les cahiers du centre de morphologie mathématique de Fontainebleau, Paris.

Régaldo-Saint Blancard, P., 1996. - Exploration archéologique de la place de la Bourse à Bordeaux. Revue archéologique de Bordeaux, tome LXXXVII, p. 39-62.

Régaldo-Saint Blancard, P., 2004 [2009]. - Le port antique de Bordeaux : bilan et nouvelles hypothèses, in Archéologie et environnement dans la Méditerranée antique (colloque 2004), Droz, 2009, p. 221-246.

RéGaldo-Saint BlanCARD, P., 2005. - Navigeram per portam : une nouvelle lecture des données archéologiques anciennes sur le port antique de Bordeaux, Revue archéologique de Bordeaux, tome XCVI : 99-128.

Régaldo-Saint Blancard, P., 2008. - Bordeaux, îlot Sud-Ouest : évaluation du potentiel archéologique, Rapport SRA.

SIREIX, C. (dir.), 1995. - Bordeaux, Cité Judiciaire, Rapport SRA d'Aquitaine, Bordeaux.

SireIX, C. (dir.), 2008. - La Cité Judiciaire : un quartier suburbain de Bordeaux antique, Aquitania, suppl. 15.

Thierry, P. et Breysse, D., avec la collaboration de E. Vanoudheusden, A. Marache, S. Dominique, B. Rodière, B. Bourgine, P. Régaldo-Saint Blancard, Ch. Piette, F. RIVET, R. FABRE, 2006. - Le projet RIVIERA : Risques en ville : Equipements, Réseaux, Archéologie, Rapport final BRGM/ RP-55085-FR.

WACKERNAGEL, H., 2003. - Multivariate Geostatistics: an introduction with applications, Springer-Verlag, Berlin, $3^{\text {rd }}$ edition. 
